RESEARCH PAPER

\title{
Feel and touch: a haptic mobile game to assess tactile processing
}

\author{
Ivonne Monarca, Monica Tentori, Franceli L. Cibrian
}

Published: 30 November 2021

\begin{abstract}
Haptic interfaces have great potential for assessing the tactile processing of children with Autism Spectrum Disorder (ASD), an area that has been under-explored due to the lack of tools to assess it. Until now, haptic interfaces for children have mostly been used as a teaching or therapeutic tool, so there are still open questions about how they could be used to assess tactile processing of children with ASD. This article presents the design process that led to the development of Feel and Touch, a mobile game augmented with vibrotactile stimuli to assess tactile processing. Our feasibility evaluation, with 5 children from 3 to 6 years old, shows that children accept vibrations and are able to use the proposed vibrotactile patterns. However, it is still necessary to work on the instructions to make the game dynamic clearer and rewards to keep the attention of children. We close this article by discussing future work and conclusions.
\end{abstract}

\section{Keywords:}

Autism spectrum disorder; Tactile processing; Haptic interfaces; Vibrotactile patterns.

\section{Introduction}

The use of screening tools (i.e., tests, examinations or other procedures of easy and quick application) during childhood is the key to detect children who show signals of ASD and who need to be referred to a specialist to obtain a complete diagnosis and receive an intervention to help them improve their skills $[4,7]$. However, most screening tools for Autism Spectrum Disorder (ASD) are based on questionnaires that parents or caregivers must answer [8] and on psychometric tests based on observation and highly interpreted by the specialists who apply the tests. This makes screening subjective, and misinterpretation can affect the possibility of obtaining a timely diagnosis. In addition, most of the questions in these tools are focused on communication and socialization skills, leaving aside sensory processing (Kanne,

Monarca, Ivonne., Tentori, Monica.

CICESE

Ensenada, Mexico

monarca@cicese.edu.mx,mtentori@cicese.mx

Cibrian, Franceli L

Chapman University

Orange, United States

cibrian@chapman.edu

${ }^{1}$ Tools that transmit information through touch.
2018). About $90 \%$ of people with ASD have atypical sensory processing [18], and those differences can be a potential marker of ASD [18].

Until now, tactile processing has been little explored due to the lack of tools to assess it. [20]. However, with the growth of haptic interfaces ${ }^{1}$, there is an untapped potential to understand and assess the tactile processing of individuals with ASD and uncover haptic digital markers ${ }^{2}[14]$

Haptic interfaces emulate tactile or kinetic properties of an object. Haptic interfaces could be: (1) passive, use spatial or material features as a stimulus, such as textures; or (2) active, use actuators as a stimulus, such as vibration motors $[12,13,19]$

Active haptic interfaces are becoming more common in our lives as the devices we used every day incorporate haptic feedback mostly in the form of vibrotactile patterns -a function of intensity, rhythm, and sharpness representing the shape of a vibratory waveform. For example, a smartphone may vibrate to notify users of a new message.

Research on active haptic interfaces to support the care of individuals with ASD has shown that there is a potential to use them as a therapeutic intervention [2,5] and learning tools [15,23]. The literature has shown that there are differences between children with ASD and neurotypical children when processing vibrations [16]; however, little has been said if haptic interfaces can be used to assess differences in tactile processing in children with ASD. There is limited research using haptic robots $[10,11]$ this aim; but open questions remain to demonstrate the potential of other haptic interfaces in this domain and understand how vibrations affect children's gestures when they interact with a haptic interface. We hypothesize that Haptic interfaces will help reveal differences between children with ASD and neurotypical children that can be used in ASD screening.

In this paper, we describe the design of Feel and Touch, a mobile haptic game augmented with vibrotactile patterns to assess tactile processing; the contributions of this paper are:

- A prototype of a mobile haptic game that appropriately encourage interaction of children with ASD.

- A set of game activities to augment the capabilities of the proposed vibrotactile patterns.

\section{Related work}

Haptic interfaces for children have been used mostly as therapeutic or learning tools. In terms of learning, research has

\footnotetext{
${ }^{2}$ Measurements used to quantify physiological and behavioral data collected through digital devices.
} 
shown that haptic interfaces have the potential to improve children's reading experience by augmenting stories with vibrations [23] and creating associations of haptic sensations with semantic meanings [24]. Moreover, haptic interfaces have been explored to help children acquire writing skills $[9,15]$

In terms of therapy, haptic interfaces have been used to support children with vision impairments to play games with a gamepad [17]. For children with ASD, the use of vibration using gamepads ease emotion recognition and reduce stereotypical behaviors [2].

More relevant to our project is CARBO, a robot with tactile stimulation to support sensory integration therapy for children with developmental disorders [11]. The study involved 18 children with Attention-Deficit/Hyperactivity Disorder (ADHD) and 1 child with ASD. The study shows that depending on the disorder, children respond differently to tactile stimuli, suggesting that haptic interfaces could be used to assess sensory processing. Similarly, haptic robots have been used to assess tactile processing [10]. A study with 6 children with ASD and 6 neurotypical children who played a game using a haptic robot, with which children could feel the "heaviness" of a ball. The results indicate that the "heaviness" of the ball affected the performance of children with ASD.

These works have shown that children can to interact with haptic interfaces with potential uses as a therapeutic, teaching, and assessment tools. However, there are still open questions to understand how vibrations affect children's gestures when they interact with a haptic interface and how such interaction can be encouraged with appropriate vibrotactile patterns and activities in this domain.

\section{Methods}

For 4 months, we follow an iterative methodology based on the user-centered design philosophy. First, we conducted a literature review to compile existing active haptic interfaces, vibrotactile patterns, and interaction gestures.

Then, we conducted a session with children and IHC experts to find out what gestures they associate with vibrations (Table 1). For this session, we first asked the participants if they knew what vibration is, and then we did brainstorming sessions; we saved the ideas in a document to be discussed and to make a match between gestures and vibrations.

To involve the specialists in the design process, we conducted 5 design sessions to get the design of the game (Table 1). These sessions involved experts in IHC, neurotypical children, preschool teachers, and a special education teacher (Table 1). The dynamics of the sessions were as follows: first, we explained the context of the study to participants, then we conducted brainstorming, and discussion of potential ideas took place; finally, low-fidelity prototypes were proposed, and we discussed the advantages and disadvantages of the prototypes. The data collected during the design sessions were analyzed and materialized as sketches and storyboards.

\section{Results}

The results are divided into two sections: (1) gestures and vibrotactile patterns and (2) description of Feel and Touch, a haptic mobile game augmented with vibrotactile stimuli to assess tactile processing.
Table 1. The table show the participants involved on participatory session, the objective and result for each session

\begin{tabular}{|l|l|l|l|}
\hline Session & Participants & Objective & Result \\
\hline 1 & $\begin{array}{l}4 \text { HCI } \\
\text { experts } \\
5 \\
\text { neurotypical } \\
\text { children }\end{array}$ & $\begin{array}{l}\text { Get a list of } \\
\text { gestures that } \\
\text { are associated } \\
\text { with vibration }\end{array}$ & $\begin{array}{l}\text { List of gestures } \\
\text { that the } \\
\text { participants as- } \\
\text { sociated with } \\
\text { vibration }\end{array}$ \\
\hline 2 & $\begin{array}{l}1 \text { HCI } \\
\text { expert } \\
2 \\
\text { neurotypical } \\
\text { children } \\
2 \text { pre-school } \\
\text { teachers } \\
1 \text { special } \\
\text { education } \\
\text { teacher }\end{array}$ & $\begin{array}{l}\text { Get an } \\
\text { association } \\
\text { between } \\
\text { gestures and } \\
\text { vibrotactile } \\
\text { patterns. }\end{array}$ & $\begin{array}{l}\text { Get the low- } \\
\text { prototype. } \\
\text { and vibrotactile } \\
\text { patterns. }\end{array}$ \\
\hline $\begin{array}{l}3 \text { HCI } \\
\text { expert }\end{array}$ & $\begin{array}{l}\text { Low-fidelity } \\
\text { prototype } \\
\text { Association }\end{array}$ \\
\hline $3,4,5$ & $\begin{array}{l}\text { Get the } \\
\text { expert } \\
\text { the game } \\
\text { designer }\end{array}$ & $\begin{array}{l}\text { Get the } \\
\text { graphic design } \\
\text { of the game }\end{array}$ & $\begin{array}{l}\text { Character and } \\
\text { scenario design }\end{array}$ \\
\hline 6 & game \\
\hline
\end{tabular}

\subsection{Gestures and vibrotactile patterns}

We obtained a list of gestures from the gesture session that experts and children associated with vibration. From that list, we selected the gestures that children from 3 to 5 years old can perform [3]: tap and drag. For each gesture, we associated a vibrotactile pattern. In particular, we associated the tap gesture to a flat pattern and then the drag gesture with a ramp pattern. (For more details, see Appendix A)

\subsubsection{Flat vibration pattern}

For the flat vibration patterns, we define $80 \%$ from the maximum intensity available within the motors used on a smartphone. We selected 3 rhythms for the flat vibration, based on those already reported in the literature $[6,21,22]$, slow (1 second between each vibration), medium (500 milliseconds between each vibration), and quick (250 milliseconds between each vibration). In our design, we defined 25 pulses for each flat vibration, based on the maximum number of stimuli used in a vibrotactile battery to assess the tactile performance of children with ASD [16].

\subsubsection{Ramp vibration pattern}

For the ramp vibration patterns, we selected both ascending and descending scales of intensity. In our design, the maximum intensity is $100 \%$, and the minimum intensity is $40 \%$ available within the motors used on a smartphone. The ascending ramps range from 40 to $100 \%$ intensity, and the descending ramps range from 100 to $40 \%$. We defined a pattern of 25 ramps, each with a duration of 2 seconds and a separation of 1 second between vibrations. 


\subsection{Feel and touch}

From our design process, we developed Feel and Touch, a mobile haptic game augmented with vibrotactile patterns to assess tactile processing. The goal of Feel and Touch is to help a hungry spider rebuild its web destroyed in a storm mimicking the storytelling of the itsy bitsy spider nursery rhyme. To be able to eat, the spider first needs to build its web. Feel and Touch has 3 activities: build the web, feed the spider, and dancing on the web.

- Build the web. The objective of this activity is to help the spider to build its web. This level requires children to follow the rhythm of the flat vibration pattern by tapping anywhere on the screen. When the children feel a vibration, they must tap anywhere on the screen, and the spider will jump from its current location to the location of the children's tap. The spider jumping trajectory will draw a line between these two points (Figure 1). This level has 3 sub-levels: (1) slow, (2) medium, (3) quick. Each sub-level corresponds with the 3 rhythms. This activity will allow us to assess the reaction time, duration of the taps, and the amount of used force [1].

- $\quad$ Feed the spider. The objective of this activity is to feed the spider. This level requires that children drag the spider's feet while they feel the ramp vibration. When a bug falls into the web, a ramp vibration is reproduced, so the child must drag the spider's legs to guide it to the bug. When the child releases the spider's leg, the spider moves and captures the bug (Figure 2). This level has 3 sub levels: ascending ramps, descending ramps, and mixed ramps. This activity will allow us to assess reaction time, the detection threshold, force, duration of drag, and final point of the drag.

- Dance on the web. The objective of this level is that children perform tap or drag gestures on the spider's web and feel the flat or ramps vibrations. If the children make multiple taps, they will feel a flat vibration, and the spider will jump. If the children make a drag, they will feel a ramp vibration the spider will dance. With this activity, we will understand which vibrations children with ASD prefer if they either like the flat or the ramp patterns. In this activity, we will count the number of taps and drags and measure the force and the time interval between each gesture.

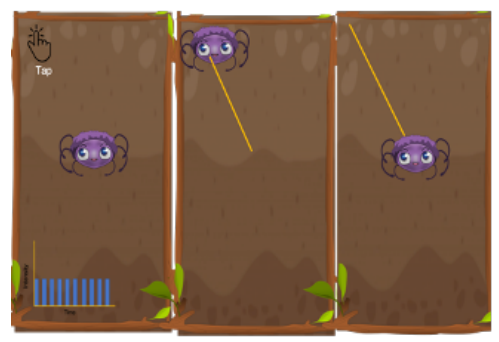

Figure 1 The images show the dynamic of build the web. The spider jumps to the point where the child taps.

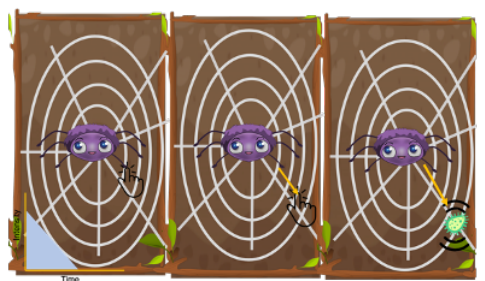

Figure 2 The image shows the dynamic of feed the spider. While the children feel the vibration, they drag the spider 'feet

\section{Feasibility evaluation of Feel and Touch}

We develop Feel and touch to run on an iPhone 11. We conducted a direct observation with 5 neurotypical children from 3 to 5 years (Table 2) old to understand whether children are able to perform the selected gestures and to respond to vibrations.

Table 2. The table show the participant demographic information

\begin{tabular}{|l|l|l|}
\hline Participant & Age & Gender \\
\hline 1 & 3 & male \\
\hline 2 & 3 & female \\
\hline 3 & 4 & male \\
\hline 4 & 5 & male \\
\hline 5 & 5 & female \\
\hline
\end{tabular}

We used the first demo of Feel and Touch and children competed in the first level of building a web and the first level of feed the spider. All children used their right hand to hold the iPhone and used their left hand to perform the gestures (Figure 3).

Each child did the two activities alone. We explained to each child the objective of the activities and gave them the same instructions.

We observed the children while they performed the activities and recorded their behavior in a document. After they finished the activities, we asked the children if they had felt vibrations before and if they could describe how the vibrations felt in the game.

From the direct observation, we found that none of the children were scared by the vibrations or made negative comments. All the children were able to perform the tap and drag gestures and responded to the vibration. For the 3-year-olds children, they needed clearer instructions to perform the tap gesture. In the first 3 attempts, the children did not tap the phone; instead, they kept one finger pressed on the screen. The rest of the children had no difficulty with the tap and drag gestures. We noticed that all the children always drag the same leg of the spider. At the end of the activities, we asked the children if they had felt vibrations before, and children answered that when their parents' phone rings, their cell phones vibrate, so children know the vibrations and are familiar with them.

These results show that it is necessary to work on the instructions to make them clearer, we also found that it is necessary to work on rewards to keep the children's attention. 


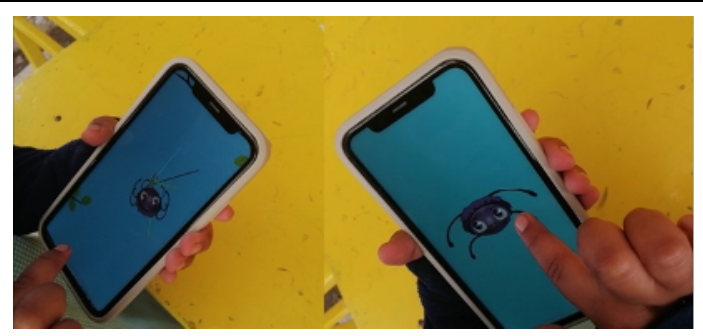

Figure 3 The image shows a child performing the activity builds the spider (left) and feeds the spider (right).

\section{Discussion: Design considerations}

As a result of the design and implementation of Feel and Touch, we identified the following design considerations:

- Use attractive visual stimuli. Haptic interfaces should incorporate attractive visualizations to help keep children engaged. For example, specialists suggested using elements that children are already familiar with and colorful animations. In Feel and Touch, we selected a spider as the main character, and the experts suggested that it should be colorful and cartoon-like.

- Use gestures that correspond to vibrotactile patterns. To interact with a haptic interface, it is necessary to associate the gestures that correspond to each haptic pattern. In Feel and Touch we associate the tap gesture to a flat vibration, and the drag gesture to a ramp, taking into account whether the gesture is discrete or continuous and the start and end characteristics of each gesture.

- Use simple instructions. Haptic interfaces should incorporate simple instructions and child-friendly language. In Feel and Touch the instructions used are "touch when you feel the vibration" and "pull one of the spider's legs while feeling the vibration".

\section{Conclusion and future work}

In this paper we present the design of Feel and touch, a mobile haptic game augmented with vibrotactile patterns to assess tactile processing. Our preliminary evaluation shows that children accept vibrations and are able to respond with proposed gestures. However, it is still necessary to work on the instructions to make the goal of each activity clearer. To complete the design process, future work is proposed to: (1) Conduct a formative study of the use of Feat to investigate whether the activities and design are appropriate (2) Conduct a study to evaluate the impact of Feat as a screening tool.

\section{References}

[1] Anna Anzulewicz, Krzysztof Sobota, and Jonathan T Delafield-butt. 2016. Toward the Autism Motor Signature: Gesture patterns during smart tablet gameplay identify children with autism. Nature Publishing Group August (2016), 1-13. https://doi.org/10.1038/srep31107

[2] Gwénaël Changeon, Delphine Graeff, Margarita Anastassova, and José Lozada. 2012. Tactile Emotions: A Vibrotactile Tactile Gamepad for Transmitting Emotional Messages to Children with Autism. In Lecture Notes in Computer Science (including subseries Lecture Notes in Artificial Intelligence and Lecture Notes in Bioinformatics). Vol. 7282 LNCS. 79-90. https://doi.org/10.1007/978-3-64231401-8\{_\}8
[3] Lucrezia Crescenzi Lanna and Mariona Grané Oro. 2019. Touch gesture performed by children under 3 years old when drawing and coloring on a tablet. International Journal of Human-Computer Studies 124 (4 2019), 1-12. https://doi.org/10.1016/j.ijhcs.2018.11.008

[4] Geraldine Dawson. 2008. Early behavioral intervention, brain plasticity, and the prevention of autism spectrum disorder. Development and Psychopathology 20, 3 (7 2008), 775-803. https://doi.org/10.1017/S0954579408000370

[5] Jie Gao, Leijing Zhou, Miaomiao Dong, and Fan Zhang. 2018. Expressive Plant. In Proceedings of the 2018 ACM International Joint Conference and 2018 International Symposium on Pervasive and Ubiquitous Computing and Wearable Computers - UbiComp '18. ACM Press, New York, New York, USA, 46-49. https://doi.org/10.1145/3267305.3267588

[6] Nava Haghighi, Nathalie Vladis, Yuanbo Liu, and Arvind Satyanarayan. 2020. The Effectiveness of Haptic Properties Under Cognitive Load: An Exploratory Study. arXiv (5 2020). http://arxiv.org/abs/2006.00372

[7] Sophie Jullien. 2021. Screening for autistic spectrum disorder in early childhood. BMC Pediatrics 2021 21:1 21, 1 (9 2021), 1-9. https://doi.org/10. 1186/S12887-021-02700-5

[8] Stephen M. Kanne, Laura Arnstein Carpenter, and Zachary Warren. 2018. Screening in toddlers and preschoolers at risk for autism spectrum disorder: Evaluating a novel mobilehealth screening tool. Autism Research 11, 7 (7 2018), 10381049. https://doi.org/10.1002/aur.1959

[9] Younhee Kim, Zoran Duric, Naomi Lynn Gerber, Arthur R Palsbo, and Susan E Palsbo. [n.d.]. Poster: Teaching Letter Writing using a Programmable Haptic Device Interface for Children with Handwriting Difficulties. Technical Report. www.sensable.com.

[10] Ankit Koirala, Amy Van Hecke, Zhiwei Yu, Kathleen A. Koth, Hillary Schiltz, Zhi Zheng, and Hillary Schiltz. 2019. An exploration of using virtual reality to assess the sensory abnormalities in children with autism spectrum disorder. Proceedings of the 18th ACM International Conference on Interaction Design and Children, IDC 2019 (2019), 293-300. https://doi.org/10.1145/3311927.3323118

[11] Jeffrey L. Krichmar and Ting Shuo Chou. 2018. A tactile robot for developmental disorder therapy. In ACM International Conference Proceeding Series. Association for Computing Machinery. https://doi.org/10.1145/3183654.3183657

[12] Ernst Kruijff, Saugata Biswas, Christina Trepkowski, Jens Maiero, George Ghinea, and Wolfgang Stuerzlinger. 2019. Multilayer haptic feedback for penbased tablet interaction. Conference on Human Factors in Computing Systems Proceedings Chi (2019), 1-14. https://doi.org/10.1145/3290605.3300373

[13] Karon E. MacLean. 2000. Designing with haptic feedback. Proceedings - IEEE International Conference on Robotics and Automation 1, April (2000), 783-787. https://doi.org/10.1109/robot.2000.844146

[14] Sven Meister, Wolfgang Deiters, and Stefan Becker. 2016. Digital health and digital biomarkers - enabling value chains on health data. Current Directions in Biomedical Engineering 
2, 1 (1 2016), 577-581. https://doi.org/10.1515/cdbme-20160128

[15] R Palluel-Germain, F Bara, A Hillairet De Boisferon, B Hennion, P Gouagout, and E Gentaz. 2007. A Visuo-Haptic Device-Telemaque-Increases Kindergarten Children's Handwriting Acquisition. Technical Report 1.

[16] Nicolaas A. J. Puts, Ericka L. Wodka, Mark Tommerdahl, Stewart H. Mostofsky, and Richard A. E. Edden. 2014. Impaired tactile processing in children with autism spectrum disorder. Journal of Neurophysiology 111, 9 (5 2014), 18031811. https://doi.org/10.1152/jn.00890.2013

[17] Roope Raisamo, Saija Patomäki, Matias Hasu, and Virpi Pasto. 2007. Design and evaluation of a tactile memory game for visually impaired children. Interacting with Computers 19, 2 (2007), 196-205. https://doi.org/10.1016/j.intcom.2006.08.011

[18] Caroline E Robertson and Simon Baron-cohen. 2017. Sensory perception in autism. Nat Rev Neurosci (2017), 671-684. https://doi.org/10.1038/nrn. 2017.112

[19] José Luis Rodríguez, Ramiro Velázquez, Carolina Del-Vallesoto, Sebastián Gutiérrez, Jorge Varona, and Josué EnríquezZarate. 2019. Active and passive haptic perception of shape: Passive haptics can support navigation. Electronics (Switzerland) 8, 3 (2019), 1-12. https://doi.org/10.3390/ electronics 8030355

[20] Magdalena Romanowicz, Munis Dundar, Dulce RomeroAyuso, Sara Jorquera-Cabrera, Gemma Rodriguez-Gil, and José-Matías Triviño-Juárez. 2017. Assessment of Sensory Processing Characteristics in Children between 3 and 11
Years Old: A Systematic Review. Article 5 (2017), 1. https://doi.org/10.3389/fped.2017.00057

[21] Hasti Seifi, Kailun Zhang, and Karon E. MacLean. 2015. VibViz: Organizing, visualizing and navigating vibration libraries. In IEEE World Haptics Conference (WHC).

[22] David Ternes and Karon E Maclean. 2008. LNCS 5024 Designing Large Sets of Haptic Icons with Rhythm. Technical Report. 199-208 pages.

[23] Nesra Yannier, Ali Israr, Jill Fain Lehman, and Roberta L. Klatzky. 2015. Feel sleeve: Haptic Feedback to enhance early reading. Conference on Human Factors in Computing Systems - Proceedings 2015-April (2015), 1015-1024. https://doi.org/10.1145/2702123.2702396

[24] Yudong Zhang, Zhengchao Dong, Preetha Phillips, Shuihua Wang, Genlin Ji, Jiquan Yang, and Ti-Fei Yuan. 2015. Detection of subjects and brain regions related to Alzheimer's disease using 3D MRI scans based on eigenbrain and machine learning. Frontiers in Computational Neuroscience 9 (6 2015), 66. https://doi.org/10.3389/fncom.2015.00066

\section{Appendix A}

Table A. The table shows the relationship between gestures and vibrotactile patterns, as well as the intensity values for each pattern.

\begin{tabular}{|c|c|c|c|c|c|c|c|c|}
\hline Gesture & $\begin{array}{l}\text { Vibrotactile } \\
\text { pattern }\end{array}$ & $\begin{array}{l}\text { Description } \\
\text { vibrotactile } \\
\text { pattern }\end{array}$ & $\begin{array}{l}\text { Start of } \\
\text { the } \\
\text { gesture }\end{array}$ & Trajectory & $\begin{array}{l}\text { End of the } \\
\text { gesture }\end{array}$ & $\begin{array}{l}\text { Example } \\
\text { vibrotactile } \\
\text { pattern }\end{array}$ & $\begin{array}{l}\text { Intensity of } \\
\text { vibrotactile } \\
\text { pattern }\end{array}$ & $\begin{array}{l}\text { Rhythms and type of } \\
\text { ramp }\end{array}$ \\
\hline Tap & Flat & $\begin{array}{l}\text { The intensity } \\
\text { remains the } \\
\text { same over time }\end{array}$ & $\begin{array}{c}\text { Vibration } \\
\text { with a } \\
\text { default } \\
\text { intensity }\end{array}$ & $\begin{array}{c}\text { No } \\
\text { trayectory }\end{array}$ & No vibration & & $80 \%$ & $\begin{array}{l}\text { Slow ( } 1 \text { second between } \\
\text { each vibration) } \\
\text { medium ( } 500 \text { milliseconds } \\
\text { between each vibration) } \\
\text { quick ( } 250 \text { milliseconds } \\
\text { between each vibration). }\end{array}$ \\
\hline
\end{tabular}

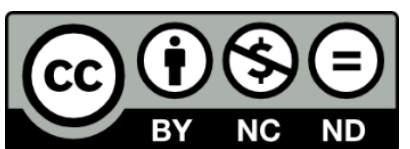

(C) 2021 by the authors. This work is licensed under the Creative Commons AttributionNonCommercial-NoDerivatives 4.0 International License. To view a copy of this license, visit http://creativecommons.org/licenses/by-nc-nd/4.0/ or send a letter to Creative Commons, PO Box 1866, Mountain View, CA 94042, USA. 